\title{
Contribución al servicio de la mejora de la salud infantil: Experiencias de campo realizada durante ocho años por estudiantes y docentes de parasitología en diferentes zonas rurales de Honduras
}

\section{Contribution to the service of improving child health: Field experiences carried out for eight years by parasitology students and teachers in different rural areas of Honduras}

\section{Introducción}

El departamento de Parasitología de la Escuela de Microbiología desde el año 2011 hasta el 2019, llevó a cabo un proyecto de vinculación universidad sociedad con el título "Prevalencia de parásitos intestinales en niños escolares de zonas rurales de Honduras". El propósito de este proyecto, además, de cumplir con las funciones del docente universitario de investigación, docencia y vinculación, fue el de realizar procesos académicos los cuales permita a los estudiantes construir su conocimiento en contextos reales que los sitúen frente a los problemas de salud de nuestra población en el que se incluye también a los actores sociales de las comunidades.
Este proyecto fue iniciado en el año 2011 bajo la coordinación de la doctora Doris Quan, quien se esmeró para que los docentes del departamento de Parasitología participaran de estas actividades de vinculación, además, motiva a los estudiantes para dar un servicio a la comunidad como se muestra en la Figura 1 y como muy acertadamente se afirma, "en nuestro trabajo con estudiantes en procesos de extensión hemos podido constatar que estos procesos mueven, conmueven e interpelan a los estudiantes en su rol como universitarios y futuros profesionales". (Tommasino \& Cano, 2016, pág. 15).

Las unidades académicas del área de la salud, como la nuestra,

Autores: ${ }^{1}$ María Mercedes Rueda Carol Anahelka Rodriguez Wendy Valladares Wilfredo Sosa Maritza Canales

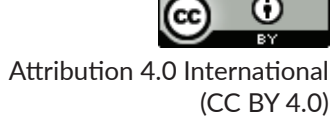

Recibido: 20 de septiembre del 2020. Aceptado: 11 de octubre del 2020

${ }^{1}$ Profesora universitaria de la Escuela de Microbiología de la Universidad Nacional Autónoma de Honduras (UNAH), maria.rueda@unah.edu.hn 


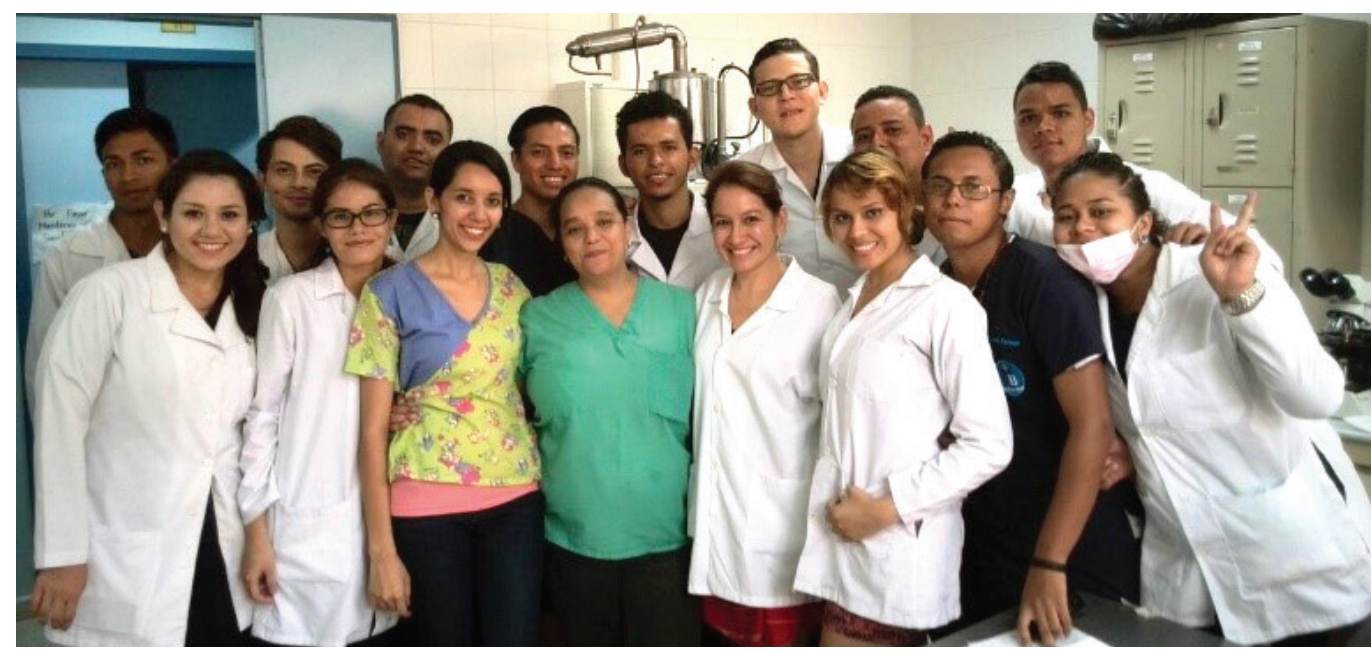

Figura 1. Grupo de estudiantes junto con cuatro docentes del departamento de Parasitología, viaje de campo en Tela, Atlántida.

promueven la transferencia de conocimientos para lograr cambios que repercutan en el bienestar de las comunidades, a través de la promoción de cambios de hábitos saludables para la prevención de enfermedades, en este caso, de parasitismo intestinal. Además, docentes y estudiantes aplican técnicas para el diagnóstico de estas infecciones en niños y proveen al médico o enfermera de los centros de salud un reporte parasitológico para que sean tratados con medicamentos específicos según el parásito encontrado.

Dentro de las infecciones más prevalentes en el mundo se encuentran las producidas por parásitos intestinales, por helmintos y protozoos, constituyendo una gran carga para la salud pública, sobre todo en la población infantil de áreas rurales marginadas. La OMS estima que existen 270 millones de niños en edad preescolar y más de 600 millones de niños en edad es- colar viviendo en áreas en las cuales los helmintos y protozoos provocan altas tasas de transmisión $y$ que urgen de intervenciones. (WHO, 2012).

Los niños de edad preescolar y escolar son más susceptibles a estas infecciones por su sistema inmunológico inmaduro, sus actividades lúdicas, actividades agrícolas o en granjas que les permite estar en contacto con suelo o animales.

Las consecuencias de estas infecciones en los menores son: retraso en el crecimiento, deterioro de la función cognitiva, avance educativo limitado y futuras perspectivas económicas comprometidas. (Barry, Simon, Mistry, \& Hotez, 2013).

Las infecciones repetidas a edades tempranas afectan negativamente el desarrollo normal de un individuo, este tipo de infecciones son frecuentes en comunidades pobres, donde no hay acceso a 
agua de calidad para consumo diario, existe además mal saneamiento ambiental e higiene básica inadecuada.

El control de estas infecciones es posible cuando se cuenta con políticas de salud sostenibles como las campañas de desparasitación masiva. En Honduras existe desde el año 1998 el programa de "Escuelas Saludables" que actualmente lidera la Secretaría de Salud en asociación con la Secretaría de Educación y el Programa Ampliado de Inmunización (PAI) y otros colaboradores, para dar cobertura a los niños en edad preescolar y escolar del sistema público de educación de nuestro país, ofreciendo a los ni- ños dos rondas de desparasitación anual. Pese a esto aún persisten estas infecciones con prevalencias mayores al $20 \%$ y en algunos sitios puntuales con porcentajes arriba de $50 \%$. (Matamoros, Schultz, Fontecha, \& Sanchez, 2017).

El control de las infecciones de helmintos trasmitidos por el suelo en niños es una prioridad a nivel mundial, en el año 2001, la OMS recomendó que para reducir la prevalencia de estas infecciones se debe al menos cubrir en un $75 \%$ a la población en riesgo de zonas endémicas (OMS, 2011). Para esto, se necesita además monitoreo parasitológico y evaluación de las estrategias.

\section{Contenido}

\section{Procedimientos}

* A través de viajes de campo realizados a diferentes comunidades rurales en diferentes zonas de Honduras para la cuales se cuenta con el apoyo y bajo convenio establecido con Global Brigades. También por iniciativa de algún estudiante de la Carrera de Microbiología participaron del proyecto que tenían algún familiar director o maestro en alguna escuela rural o porque alguno de ellos, solicitaron se visitara la escuela de su comunidad de origen en la que habían estudiado, además algunos alcaldes se acerca- ron solicitando el proyecto para sus comunidades o docentes que ofrecieron la actividad en algunas escuelas.

Para esto fue necesario obtener el permiso de las autoridades escolares, padres de familia y de cada menor participante.

En la Figura 2; se muestra una de las reuniones previas en donde se les explicaba el proyecto y se les solicitaba firmar el consentimiento informado si ellos aceptan entonces se les daban instrucciones y el material para la toma de muestras de materia fecal. 


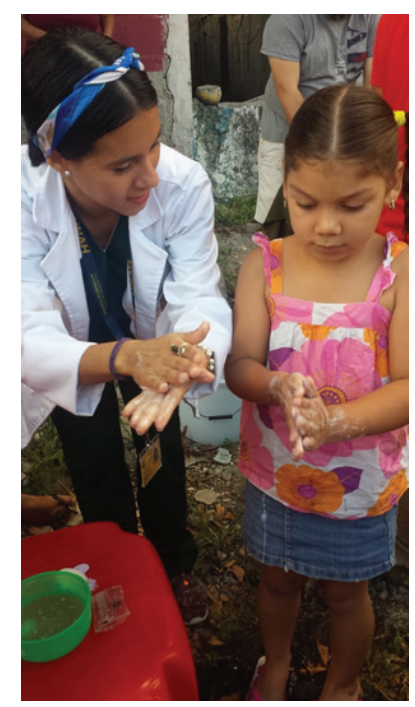

Figura 3. Estudiante enseñando a los niños el correcto lavado de manos con agua y jabón.

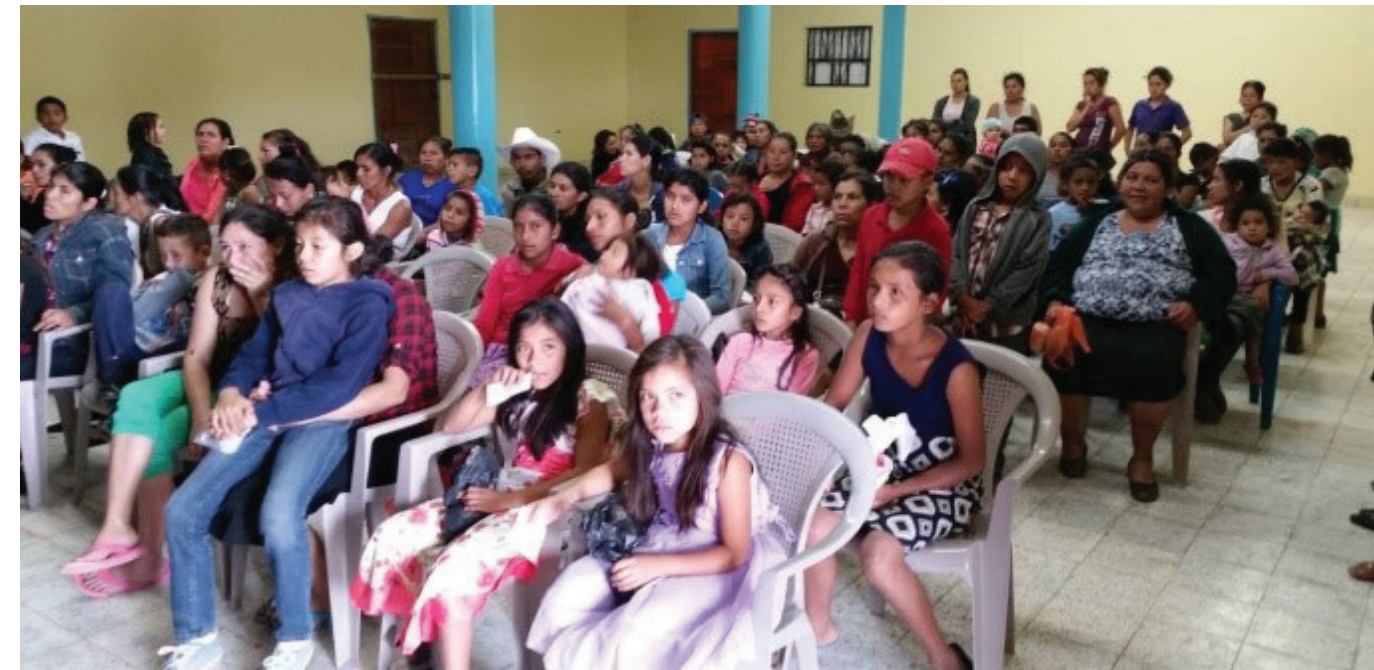

Figura 2. Reunión con miembros de la comunidad para explicarles el proyecto y firma de consentimiento informado.

${ }^{*}$ A partir de estos trabajos de campo se obtuvo como resultado que se firmara como contraparte una carta de convenio interinstitucional entre Global Brigades y la UNAH a través de la Dirección de Vinculación Universidad Sociedad, que además les brindó a los estudiantes de la carrera la oportunidad de compartir experiencias con estudiantes y profesionales de la salud de Estados Unidos que vienen a realizar brigadas médicas, odontológicas, mejora de viviendas y construcción de unidades de almacenamiento y tratamiento de agua.

En relación con la práctica de los estudiantes de Microbiología fueron entrenados en el adecuado uso de métodos coproparasitológicos de concentración para aumentar la sensibilidad en el diagnóstico (método de Kato Katz para la evaluación de helmintos transmitidos por el suelo, méto- do de formol acetato de etilo para evaluación de protozoos intestinales).

* En cuanto a la experiencia, enseñanza aprendizaje, los estudiantes analizan la epidemiología, el entorno social y cultural asociados a estas infecciones; además de lograr en ellos un liderazgo técnico al poner en práctica sus destrezas en el procesamiento de muestras a través de métodos sensibles y confiables de análisis coproparasitológico, el diagnóstico microscópico, además de trabajar bajo estrictos controles de calidad. También impartieron charlas educativas sobre[JA1] [MMRH2] higiene y prevención en salud como el correcto lavado de manos con agua y jabón como se muestra en la Figura 3, lavado de frutas antes de comer, la importancia de la correcta disposición de excretas, el baño diario entre otros. 


\section{Actividades principales}

\section{Población involucrada en el desarrollo de actividades}

Las actividades fueron desarrolladas personas de comunidades rurales visitadas, por estudiantes de las asignaturas de Protozoología MB-076, Helmintología MB107, Pasantía de Parasitología MB-240, tesistas de pregrado y el personal docente del departamento de Parasitología de la Escuela de Microbiología, Facultad de Ciencias de la Universidad Nacional Autónoma de Honduras .

- Criteritos de selección de los participantes

El estudio incluyó en mayor porcentaje muestras de heces de escolares de ambos géneros en edades que oscilaban de cuatro hasta quince años, sin embargo; en algunos casos particulares por solicitud personal se realizaron también algunas muestras de población adulta de la comunidad y personal docente o administrativo de la escuela de los lugares intervenidos.

- Descripción de Instrumentos y/o técnicas

En la Figura 4 se muestran las actividades desarrolladas las que se organizaron en diferentes fases.
Cabe mencionar que el componente de ética es indispensable, ya que, los participantes fueron menores de edad; se realizaron primero sesiones informativas sobre los objetivos del estudio, toma de muestras, riesgos y beneficios de participar en el proyecto. Se especificó que el objetivo principal es mejorar la calidad de vida de la población infantil mediante el diagnóstico y tratamiento de los escolares con parásitos intestinales. Los estudiantes de la carrera impartieron charlas sobre parásitos intestinales, lo que estas infecciones producen en un niño, cómo se transmiten y formas de prevención y control. Posteriormente a cada padre se le solicitó que firmaran consentimientos y se obtuvo también el asentimiento de los menores.

Se realizaron vistas preliminares a las escuelas para conocer a los directores y maestros y explicarles detalladamente el proyecto, y si estaban de acuerdo se firmó un documento de aceptación por parte de cada director. Únicamente se intervinieron escuelas con su autorización firmada. 


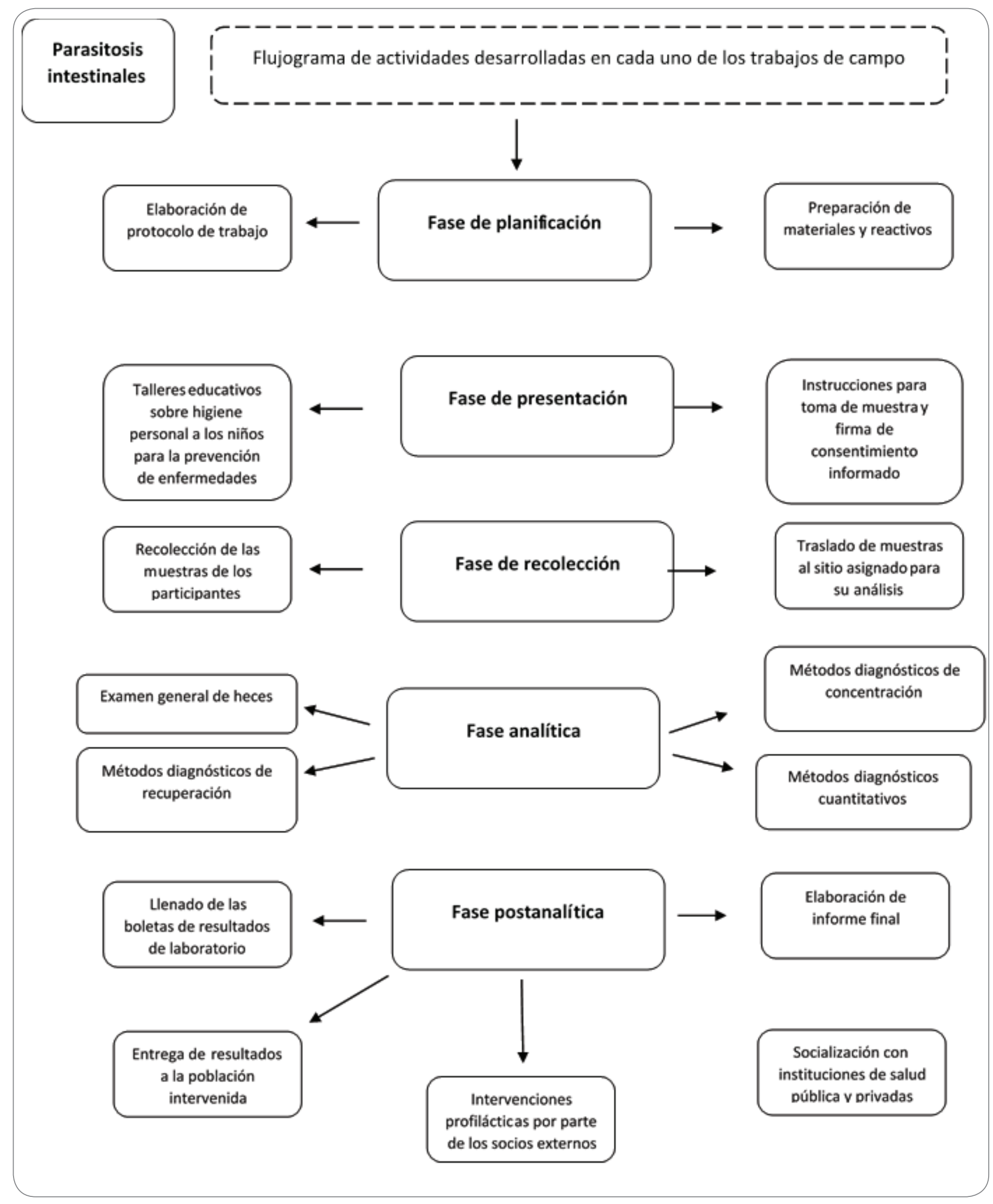

Figura 4. Flujograma de las actividades desarrolladas en los trabajos de campo. 
Tabla 1. Departamentos, comunidades y población infantil involucradas.

\begin{tabular}{|lcc|}
\hline Departamento & $\begin{array}{c}\text { No. } \\
\text { Comunidades }\end{array}$ & $\begin{array}{c}\text { No. } \\
\text { Participantes }\end{array}$ \\
\hline Valle & 6 & 481 \\
Francisco Morazán & 29 & 1697 \\
El Paraíso & 9 & 753 \\
Olancho & 2 & 118 \\
Santa Bárbara & 1 & 95 \\
Cortes & 1 & 28 \\
Choluteca & 6 & 484 \\
Copan & 1 & 111 \\
Atlántida & 2 & 78 \\
Comayagua & 1 & 85 \\
La Paz & 1 & 24 \\
\hline Total & $\mathbf{6 0}$ & $\mathbf{4 0 8 2}$ \\
\hline
\end{tabular}

Tabla 2. Características de la población infantil con infecciones intestinales parasitarias

\begin{tabular}{|c|c|}
\hline Características & Porcentaje (\%) \\
\hline \multicolumn{2}{|l|}{ Grupo de Edades } \\
\hline 0-4 años & $10 \%$ \\
\hline 5-15 años & $88 \%$ \\
\hline Mayor 15 años & $2 \%$ \\
\hline \multicolumn{2}{|l|}{ Género } \\
\hline Femenino & $48 \%$ \\
\hline Masculino & $52 \%$ \\
\hline \multicolumn{2}{|l|}{ Nivel de escolaridad } \\
\hline Pre-escolar & $8 \%$ \\
\hline Escolar & $83 \%$ \\
\hline Otros & $9 \%$ \\
\hline
\end{tabular}

\section{Registro de ejecución}

Se realizaron durante ese tiempo evaluaciones en 60 comunidades de doce departamentos del país como se presenta en la Tabla 1, se realizaron un total de cuatro mil 82 análisis coproparasitológicos de niños en edad escolar, los cuales presentaron una prevalencia por infecciones parasitarias intestinales de un $67 \%$.

En la Tabla 2 se puede observar que en la población intervenida los niños de entre los cinco a quince años y los varones en edad escolar, presentaron mayor prevalencia de infecciones por parásitos intestinales.

En la Figura 5 se muestra la prevalencia por cada parásito encontrado. Dentro de estos la mayoría fueron los protozoos intestinales comensales siendo Blastocystis spp. el que se presentó con más frecuencia, con un $30 \%$ de prevalencia. Es un organismo cuyo potencial patogénico es controversial y se encuentra frecuentemente en la materia fecal de personas sanas que no presentan síntomas digestivos, a veces se encuentra en personas con diarrea, dolor abdominal u otros problemas gastrointestinales. (del Coco, Molina, Basualdo, \& Córdoba, 2017).

Estos organismos comensales son de origen intestinal se adquieren por ingerir alimentos, agua o fómites contaminados con materia fecal.

Entre los protozoarios patógenos, el flagelado intestinal no invasivo Giardia intestinalis presentó una prevalencia de $11 \%$, causa alrededor de 280 millones de casos de diarrea a nivel mundial, pueden presentarse casos asintomáticos o manifestarse como mala digestión, mala absorción, desequilibrio electrolítico y se pueden dar casos agudos o desarrollar cronicidad. (Ma'ayeh, y otros, 2017).

En relación con las infecciones por helmintos la más frecuentes 


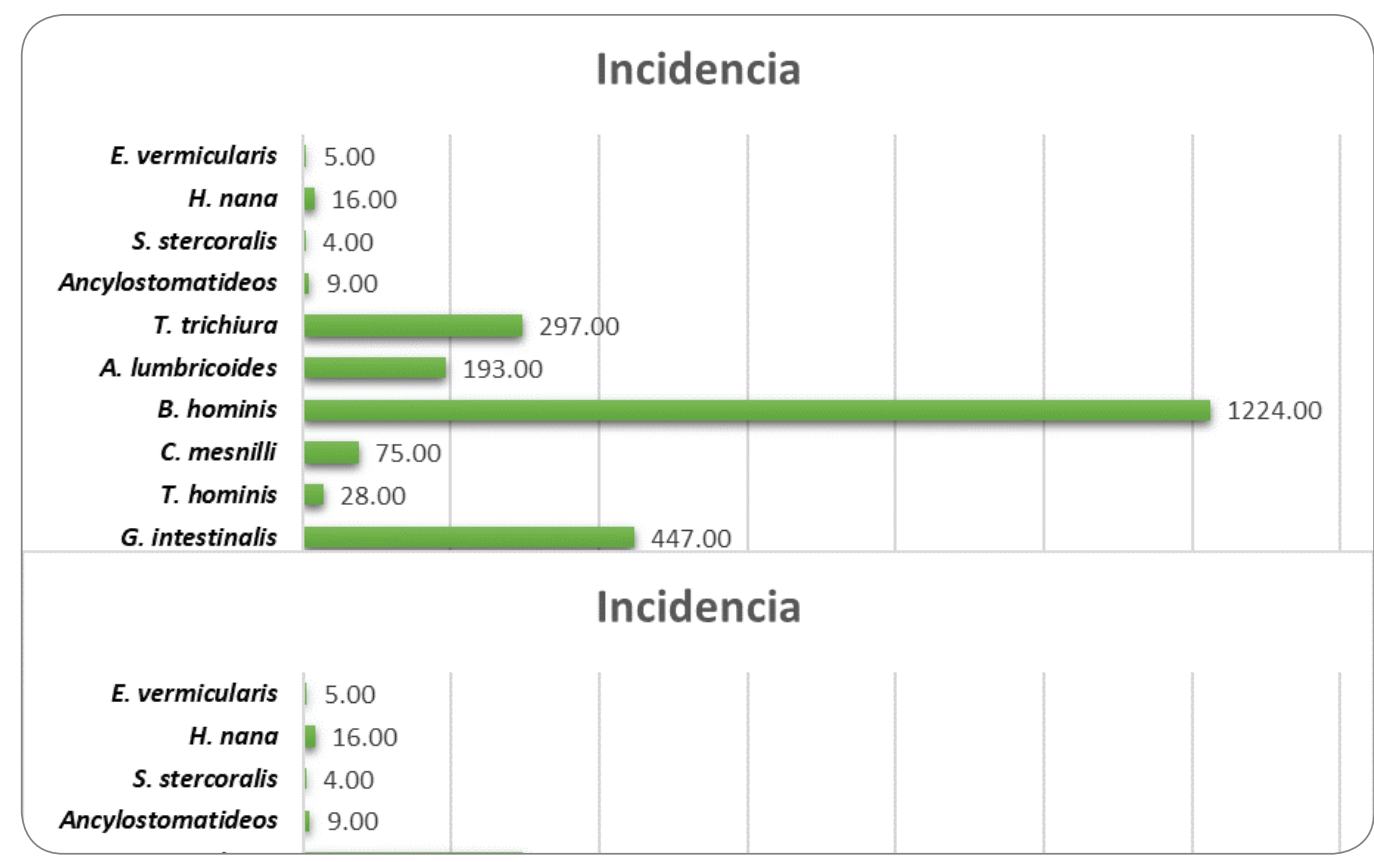

Figura 5. Características de la población infantil con infecciones intestinales parasitarias

fueron por Trichuris trichiura $7 \%$, seguida por Ascaris lumbricoides con $5 \%$, los cuales forman parte del grupo de los geohelmintos, como se ha mencionado antes, pueden producir una morbilidad asociada a la cantidad de gusanos presentes en el intestino. La intensidad alta de estas infecciones causa deterioro en el crecimiento físico, desarrollo cognitivo, defi- ciencia de micronutrientes y desarrollo de anemia por deficiencia de hierro. (Gabrie, Rueda, Rodríguez, Canales, \& Sanchez, 2016).

La baja prevalencia mostrada en las infecciones por helmintos transmitidos por el suelo puede deberse a las campañas de desparasitación masiva realizadas por la Secretaría de Salud.

\section{Materiales y equipos especiales}

\section{Examen directo de la materia fecal}

Fundamento: se basa en preparaciones húmedas directas de las muestras de heces empleando solución salina y Lugol, que brindan condiciones adecuadas para que los microorganismos mantengan sus características biológicas estables y lograr la fijación, e identificación de parásitos intestinales, respectivamente. El análisis de las heces es una de las técnicas diagnósticas de uso frecuente en medicina. 
Tabla 3 Intensidad de la Infección en especie de helmintos transmitidos por el suelo.

\begin{tabular}{cccc} 
& \multicolumn{4}{c}{$/ /$ Tomado de (Montresor, Crompton, Gyorkos, \& Savioli, 2002) } \\
\hline HTS & \multicolumn{4}{c}{ Umbral de intensidad de infección } \\
\hline & Leve & Moderada & Severa \\
Ascaris lumbricoides & $1-4,999$ HPG* & $5,000-49,999$ HPG & $\geq 50,000$ HPG \\
Trichuris trichiura & $1-999$ HPG & $1,000-9,999$ HPG & $\geq 10,000$ HPG \\
Ancylostomatídeos & $1-1,999$ HPG & $2,000-3,999$ HPG & $\geq 4,000$ HPG \\
\hline
\end{tabular}
*HPG: huevo por gramo de heces

\section{Kato-Katz}

Fundamento: es un método de concentración cualitativo y cuantitativo, que tiene sensibilidad superior a otros métodos utilizados de rutina, pues utiliza una cantidad de muestra de materia fecal de $41.7 \mathrm{mg}$ comparado con $2 \mathrm{mg}$ del método directo. Es un método que lleva un proceso de aclaramiento de la muestra con glicerina, el cual permite que los huevecillos de los helmintos transmitidos por el suelo se detecten más fácilmente con el uso de una plantilla calibrada.

El conteo sistemático de huevecillos permite una estimación cuantitativa de la carga parasitaria por lo que se puede decir si una infec- ción es leve, moderada o severa como se muestra en la Tabla 3; además, de poder identificar morfológicamente los tres principales helmintos transmitidos por el suelo (Ascaris lumbricoides, Trichuris trichiura y Ancylostomatídeos) y al ser un método de concentración se pueden observar también huevecillos de otros helmintos indicando en el reporte solo su presencia sin hacer conteo (Taenia sp, Hymenolepis nana, Hymenolepis diminuta). (Gabrie, Rueda, Canales, \& Sánchez, 2012).

\section{Formol acetato de etilo:}

Fundamento: método de concentración por sedimentación basada

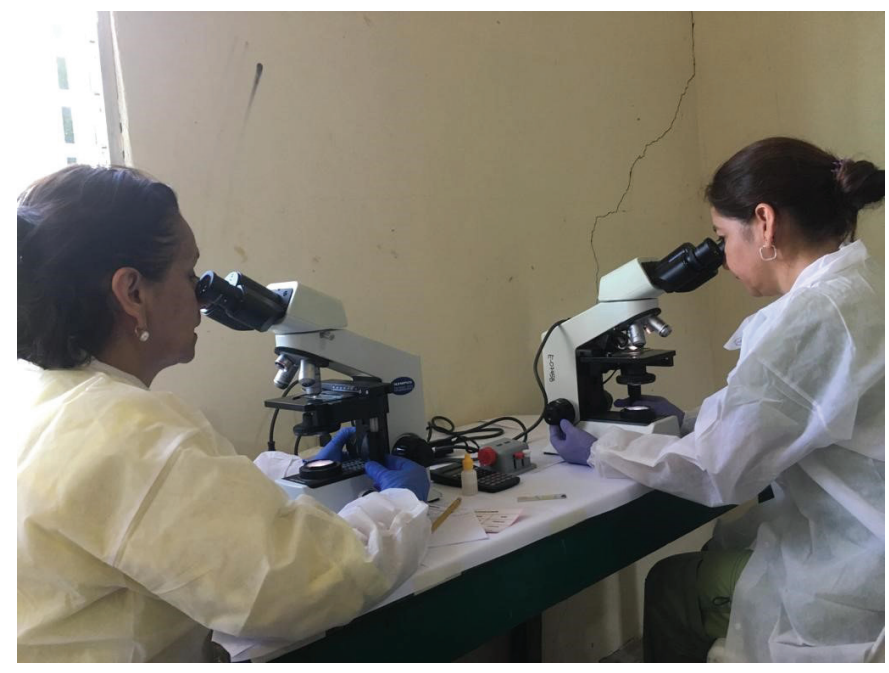

Figura 6. Control de calidad de las muestras, por parte de los docentes.

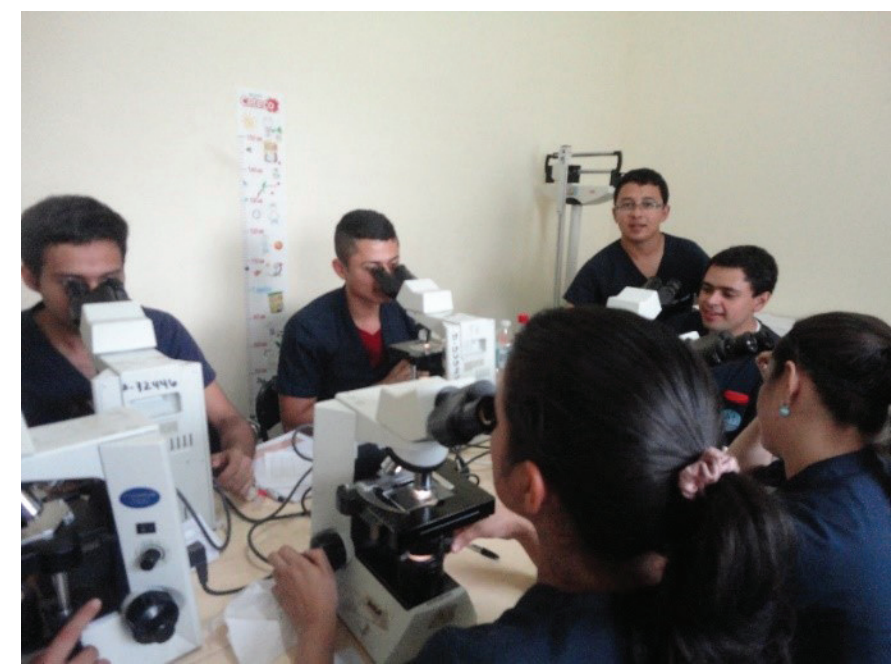

Figura 7. Estudiantes realizando microscopía para el diagnóstico coproparasitológico. 
en el uso de soluciones que permiten fijar (formalina) las estructuras parasitarias y separar (acetato de etilo) los detritos de una muestra de heces. Útil para el diagnóstico de huevos y larvas de helmintos, quistes de protozoos. (Girard de Kaminsky, 2003).

Siempre se realizan controles de calidad de todo el proceso, el cual está a cargo de los docentes como se muestra en la Figura 6.

En la Figura 7, se muestra a los estudiantes realizando el proceso de la microscopía luego de preparar las muestras por las técnicas antes mencionadas.

\section{Conclusiones}

* A lo largo de ocho años de este proyecto han pasado varias generaciones de estudiantes de Microbiología muchos de ellos ahora profesionales y algunos ahora forman parte de los docentes de nuestra Escuela, quienes han mantenido ese espíritu de empatía y solidaridad con las poblaciones necesitadas de diagnósticos laboratoriales de alta calidad; varios de ellos en su servicio social han replicado este tipo de trabajo como proyección social.

* La prevalencia de enfermedades intestinales parasitarias nos indica que se necesita de más educación y promoción en salud; que para combatirlas se deben incluir el acceso de agua de calidad para consumo, mejoramiento de la vivienda, una adecuada disposición de excretas y las rondas de desparasitación constantes, evaluadas y monitoreadas.

* Generar en los procesos de vinculación una sistematización que nos permita medir los alcances, el aprendizaje de todos los actores y sobre todo el impacto en la comunidad.

* Debemos incluir a otras áreas de la Microbiología y de otras carreras para hacer intervenciones más sólidas y que dejen mucho más beneficio para las comunidades.

* Es muy importante escuchar a la comunidad, saber cuáles son sus necesidades, apoyarles y evaluar con ellos las estrategias a implementar, fomentar el trabajo colaborativo para que los cambios que se generen sean sostenibles a lo largo del tiempo. 


\section{Reconocimientos}

Se agradece a las instituciones con las que hemos hecho alianzas para la realización de este proyecto, Global Brigades, fundación Misión Misericordia, Casa Alianza, alcaldes, directores y docentes de las escuelas, por su invaluable apoyo.

A los pobladores de las comunidades que de forma muy amable nos permitieron el desarrollo de esta actividad y que fueron anuentes para hacerles el diagnóstico a los niños.

Y a los compañeros del departamento de Parasitología, que participaron primero como estudiantes, actualmente como profesionales de la Microbiología que, con mucho entusiasmo, esfuerzo y cariño, han brindado su tiempo y sus conocimientos para la formación de los estudiantes, para la mejora de la salud infantil de los niños de Honduras.

\section{Reflexión}

A través de este tiempo han sido muchas las experiencias y aprendizajes adquiridos. En nuevas propuestas se fomentará una mejor sistematización de las actividades, realizar estrategias de vinculación innovadoras, para lograr una mayor participación y compromiso de las comunidades. Se ha comprendido que la vinculación debe ir más allá de asistir a los pobladores en un momento determinado; apoyarlos para que comprendan que los cambios que perduran solo se logran con la participación comunitaria.

Fomentar la firma de nuevos convenios que nos permitan hacer un programa de seguimiento de los procesos realizados y lograr una más efectiva medición del impacto de cada proyecto de extensión, especialmente motivar a las comunidades para su organización y empoderarlos de los procesos para que sea sostenible logrando de esta manera la mejora de la calidad de vida de los habitantes.

Crear proyectos con la colaboración de equipos interdisciplinarios, para comprender la problemática de la salud de una comunidad de una manera global. Además, invitar alumnos y docentes de postgrados pues deben surgir propuestas de líneas de investigación cuyos resultados se apliquen para beneficio de la sociedad. motivar a las comunidades para su organización y empoderarlos de los procesos para que sea sostenible 


\section{Referencias}

Barry, M. A., Simon, G. G., Mistry, N., \& Hotez, P. J. (9 de September de 2013). Global trends in neglected tropical disease control and elimination:Impact on child health. BMJ, 98(8), 635-641. doi:doi: 10.1136/archdischild-2012-302338

del Coco, V. F., Molina, N. B., Basualdo, J. A., \& Córdoba, M. A. (January-March de 2017). Blastocystis spp.: advances, controversies and future challenges. Revista Argentina de Microbiología, 49(1), 110-118. doi:https://doi. org/10.1016/j.ram.2016.08.004

Gabrie, J. A., Rueda, M. M., Canales, M., \& Sánchez, A. (2012). Utilidad del método de Kato-Katz para diagnóstico de uncinariasis: experiencia en una zona rural de Honduras, 2011. Revista Médica Hondureña, 80(3).

Gabrie, J. A., Rueda, M. M., Rodríguez, C. A., Canales, M., \& Sanchez, A. L. (2016). Immune Profile of Honduran Schoolchildren with Intestinal Parasites: The SkewedResponse against geohelmiths. Hindawi. doi:http://dx.doi. org/10.1155/2016/1769585

Girard de Kaminsky, R. (2003). Manual de parasitología métodos para laboratorios de atención primaria de salud. Tegucigalpa, Honduras.

Ma'ayeh, S. Y., Liu, J., Peirasmaki, D., Hörnaeus, K., Bergström Lind, S., Grabherr, M., Svärd, S. G. (11 de December de 2017). Characterization of the Giardia intestinalis secretome during interaction with human intestinal epithelial cells: The impact on host cells. Plos Neglected Tropical Diseases, 11(12). doi:10.1371/journal.pntd.0006120

Matamoros, G., Schultz, J., Fontecha, G. A., \& Sanchez, A. L. (2017). Persistencia de los geohelmintos en Honduras: pobreza, baja eficacia del tratamiento y potencial emergencia de resistencia parasitaria. Investigación clínica, 58(4), 393-405.

Montresor, A., Crompton, D., Gyorkos, T., \& Savioli, L. (2002). Helminth control in school-age children: a guide for managers of control programmes. Geneva: WHO.

OMS. (2011). Helminth control in school-age children A guide for managers of control programmes Seconde edition.

Tommasino, H., \& Cano, A. (enero-marzo de 2016). Modelos de extensión universitaria en las universidades latinoamericanas en el siglo XXI: tendencias y controversias. Redalyc.org(67), 7-24. Obtenido de http://www. redalyc.org/articulo.oa?id=37344015003

WHO. (2012). Deworming to combat the health and nutritional impact of soil transmitted helminths. 